\title{
Second-Harmonic Distortion in Vertical-Cavity Surface-Emitting Lasers with Lateral Loss Effects
}

\author{
P. C. Chui and S.-F. Yu
}

\begin{abstract}
The influence of lateral loss effects on the secondharmonic distortion (SHD) of vertical-cavity surface-emitting lasers is investigated theoretically. Two parameters, differential confinement factor and differential cavity loss, are defined for the consideration of self-focusing and diffraction loss. It is found that SHD increases at low modulation frequencies due to the presence of differential cavity loss, especially for lasers with small core radius.
\end{abstract}

Index Terms - Lateral loss, second-harmonic distortion, semiconductor lasers modeling, small-signal modulation, vertical-cavity surface-emitting lasers.

\section{INTRODUCTION}

$\mathbf{I}$ NTENSITY noise and chromatic fiber dispersion in analog optical communication systems are attributed to harmonic and intermodulation distortion in the amplitude modulation (AM) of semiconductor lasers. In addition, maximum usable bandwidth of the communication systems is limited by harmonic distortion [1], [2]. Therefore, devices with high linearity or small distortion, such as vertical cavity surfaceemitting lasers (VCSEL) with stable single-mode operation, are highly desired. In fact, second-harmonic distortion (SHD) of VCSEL can be much lower than that of facet emitted devices due to its high relaxation oscillation frequency [3], since SHD is inversely proportional to the square of the relaxation oscillation frequency of lasers [4].

Nonlinearity such as gain compression, spontaneous emission and longitudinal spatial hole burning have been studied extensively on the induction of SHD in semiconductor lasers [3]. In general, SHD is induced in Fabry-Perot lasers by spontaneous emission and gain compression as well as spatial hole burning at modulation frequencies less than $0.1 \mathrm{GHz}$ [2]. In particular, spontaneous emission has an influence on SHD at low bias (i.e., near threshold) but gain compression and spatial hole burning dominate at high bias level (i.e., 1.5 times threshold or above). Due to the use of Fabry Perot type resonator, similar dependency of SHD on these nonlinearities is expected in VCSEL. However, the modulation response of VCSEL may exhibit differently to that of facet emitted devices due to increased lateral losses because of its small core radius. In fact, it has been shown that lateral loss effects suppress selfsustained pulsation operation in VCSEL even with sufficient saturable absorption [5]. Therefore, lateral loss effects should

Manuscript received November 24, 1998; revised April 2, 1999. This work was supported by RGC Grant HKU7059/98E.

The authors are with the Department of Electrical and Electronic Engineering, University of Hong Kong, Pokfulam Road, Hong Kong.

Publisher Item Identifier S 1077-260X(99)05348-4. be taken into consideration in the analysis of the modulation response of VCSEL.

In this paper, the influence of lateral loss effects on the SHD of VCSEL is studied theoretically. Other nonlinearity such as spontaneous emission and spatial hole burning are ignored in the investigation as they are not of prime interest here. In Section II, the dependence of lateral loss effects on the carrier concentration under small-signal modulation is clarified. Differential confinement factor and differential cavity loss are defined in the calculation of lateral losses to take account of self-focusing and diffraction loss. In Section III, a simple rate-equation model of VCSEL is developed and SHD in the AM response of VCSEL is derived. Using this model, the values of confinement factor and cavity loss are extracted from the measured light/current curves of a fabricated VCSEL and a simulation is performed to investigate the influence of lateral loss effects on the SHD of the VCSEL. It is found that at low modulation frequencies, differential cavity loss has a much higher influence on SHD for devices with small core radius, while differential confinement factor has little effect. Brief discussion and conclusion are given in Section IV.

\section{TheORETICAL PREDiction OF LATERAL LOSS EFFECTS IN VCSEL}

\section{A. Confinement Factor and Differential Confinement Factor}

Fig. 1 shows the phenomenon of self-focusing in VCSEL. The mechanism of self-focusing can be explained as the increase in focusing of the lateral field into the core region as the refractive index inside the active layer increases. In result the lateral optical confinement factor $(\Gamma)$ of VCSEL is increased. For lasers under small signal modulation, the variation of refractive index inside the active layer is mainly due to the change of carrier concentration. Hence, the change of lateral confinement factor due to self-focusing is related to carrier concentration $N$ and can be approximated by

$$
\Gamma\left(N_{s}+\Delta N(t)\right)=\Gamma_{s}+\frac{\partial \Gamma}{\partial N} \Delta N(t)
$$

where $\Delta N$ is the small change of carrier concentration, $\Gamma_{s}$ and $N_{s}$ are the lateral confinement factor and carrier concentration, respectively, at steady state. The parameter, $\partial \Gamma / \partial N$, is defined as the differential confinement factor which describes the modulation response of lateral confinement factor. It is shown in [6] that $\Gamma_{s}$ decreases, but $|\partial \Gamma / \partial N|$ increases with the reduction of core radius. However, the sign of $\partial \Gamma / \partial N$ depends on the lateral optical confinement structure of lasers and 

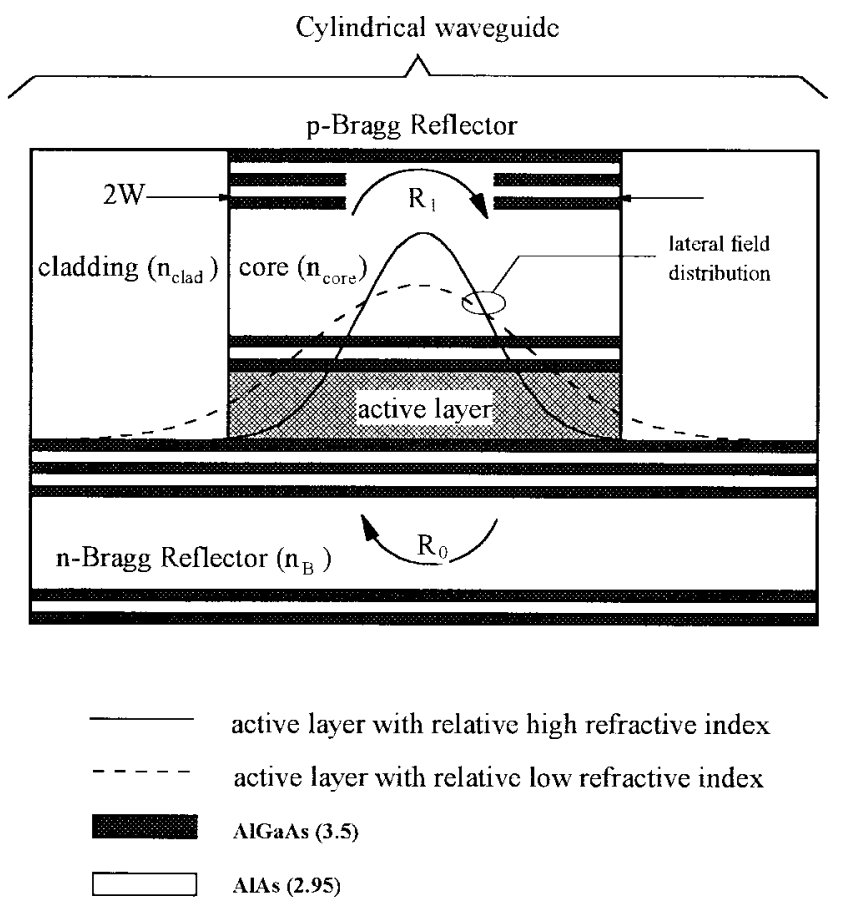

Fig. 1. Lateral distribution of optical field inside the active layer of a VCSEL. The solid line indicates that the focusing of lateral optical field into the core region due to the increase of refractive index inside the active layer.

$\partial \Gamma / \partial N$ has a negative value for VCSEL with index guiding structures.

\section{B. Cavity Loss and Differential Cavity Loss}

Fig. 2 shows the diffraction of light from a cylindrical waveguide into a Bragg reflector. The core radius of the device is $W$; the refractive indices of core and cladding regions of the cylindrical waveguide are $n_{\text {core }}$ and $n_{\text {clad }}$, respectively. The resultant angle of diffraction $\theta_{2}$, from the cylindrical waveguide (core and cladding) of effective refractive index $n_{\text {eff }}$ into the Bragg reflector with effective refractive index $n_{B}$ can be calculated from the theory of Fraunhofer diffraction and Snell's law of refraction [7]. Fig. 2(b) shows the angle of diffraction $\theta_{1}$ as light leaves the core region of a circular aperture of radius $W$. From Fraunhofer diffraction theory

$$
\theta_{1}=\sin ^{-1}\left(\frac{\lambda_{o}}{2 W}\right)
$$

where $\lambda_{\circ}$ is the wavelength in the core region. Light is further refracted to $\theta_{2}$ in the Bragg reflector. By Snell's law of refraction

$$
n_{\mathrm{eff}} \sin \theta_{1}=n_{B} \sin \theta_{2} .
$$

Substituting (3) into (2), $\theta_{2}$ can be expressed as

$$
\theta_{2}=\sin ^{-1}\left(\frac{\lambda_{o} n_{\mathrm{eff}}}{2 n_{B} W}\right) \text {. }
$$

Since the reflectivity $R$ of a Bragg reflector is a function of $\theta_{2}$ [8], (4) implies that $R$ is dependent on $n_{\text {eff }}$. For the VCSEL shown in Fig. 1, the cavity loss $\alpha$ is given by

$$
\alpha=\alpha_{m}+\frac{1}{L_{\mathrm{eff}}} \log \left(\frac{1}{\left|R_{o}\right|\left|R_{1}\right|}\right)
$$

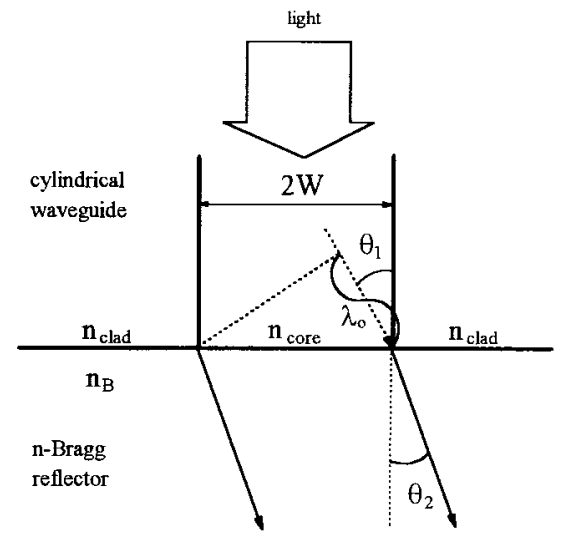

(a)

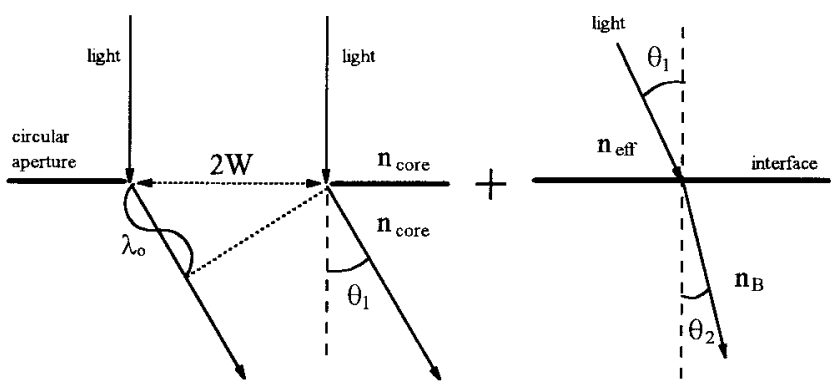

(b)

Fig. 2. The mechanism of light diffraction from a cylindrical waveguide. (a) Light diffraction through a circular aperture and refraction of light between two dielectric materials of refractive indices $n_{\mathrm{eff}}$ and $n_{B}$, respectively. (b) The explanation of light diffraction from a cylindrical waveguide using theory of Fraunhofer diffraction and Snell's Law of refraction.

where $\alpha_{m}$ is the material loss and $L_{\text {eff }}$ is the effective cavity length. $\left|R_{0}\right|$ and $\left|R_{1}\right|$ are the field reflectivity of the n- and ptype Bragg reflectors, respectively. Hence, $\alpha$ is also dependent on $n_{\text {eff }}$ as $\left|R_{0}\right|$ and $\left|R_{1}\right|$ are both functions of $n_{\text {eff }}$.

For devices under small signal modulation, any variation of $\alpha$ with small change of $n_{\text {eff }}$ can be related to $\Delta N$ by

$$
\alpha\left(N_{s}+\Delta N(t)\right)=\alpha_{s}+\frac{\partial \alpha}{\partial N} \Delta N(t)
$$

where $\alpha_{S}$ is the total cavity loss at steady state and $\partial \alpha / \partial N$ is the differential cavity loss. The value of $\alpha_{s}$ can be deduced from the steady state value of $\left|R_{0}\right|$ and $\left|R_{1}\right|$ through (5). $\partial \alpha / \partial N$ can be obtained by differentiating (5) with respect to the carrier concentration, that is

$$
\frac{\partial \alpha}{\partial N}=-\frac{1}{L_{\mathrm{eff}}}\left(\frac{1}{\left|R_{0}\right|} \frac{\partial\left|R_{0}\right|}{\partial N}+\frac{1}{\left|R_{1}\right|} \frac{\partial\left|R_{1}\right|}{\partial N}\right)
$$

where $\alpha_{m}$ and $L_{\text {eff }}$ are assumed to be independent of the carrier concentration. $\partial\left|R_{0}\right| / \partial N$ and $\partial\left|R_{1}\right| / \partial N$ are the rate of change of $\left|R_{0}\right|$ and $\left|R_{1}\right|$ with respect to the carrier concentration, respectively. The value of $\partial \alpha / \partial N$ can thus be calculated from (7) provided that $\left|R_{0}\right|$ and $\left|R_{1}\right|$ are determined. The field reflectivity $R_{0}$ can be computed from [9]

$$
R_{0} \approx \int_{0}^{\infty} \frac{\beta_{0}-H}{\beta_{0}+H} \Phi_{0} \Phi_{0}^{*} d k
$$

where $H$ is a transfer function of the Bragg reflector in $k$ space, $\beta_{0}$ and $\Phi_{0}$ are the propagation coefficients and the Hankel 
transform of the fundamental lateral mode, respectively. It must be noted that $H, \beta_{0}$, and $\Phi_{0}$ are all dependent on $n_{\text {eff }}$. $R_{1}$ can be calculated using (8) in a similar manner.

It is expected that $\left|R_{0}\right|$ (as well as $\left|R_{1}\right|$ ) decreases with the reduction of $W$ due to increased diffraction. Hence, the value of $\alpha_{s}$ increases with the reduction of $W$ as $\alpha_{s}$ is inversely proportional to $\left|R_{0}\right| .|\partial \alpha / \partial N|$ would also increase with the reduction of $W$ but the sign of $\partial \alpha / \partial N$ depends on the lateral optical confinement structure of VCSEL. For devices with index-guiding structure, $\partial \alpha / \partial N$ is negative. This can be shown by expressing (8) into a Fresnel reflectivity formula as given by [10]

$$
\left|R_{0}\right| \approx \frac{n_{B}-n_{\mathrm{eff}}}{n_{B}+n_{\mathrm{eff}}} .
$$

$\left|R_{0}\right|$ must be greater than zero and this implies $n_{B}>n_{\mathrm{eff}}$. For devices with index guiding structure, the imaginary part of $n_{\mathrm{eff}}$ is negligible such that $\partial\left|R_{0}\right| / \partial N$ can be expressed as

$$
\frac{\partial\left|R_{0}\right|}{\partial N}=\frac{-2 n_{B}}{\left(n_{B}+n_{\mathrm{eff}}\right)^{2}} \frac{\partial n_{\mathrm{eff}}}{\partial N}
$$

where $\partial n_{\mathrm{eff}} / \partial N\left(\approx n_{\text {core }} / \partial N<0\right)$ is the effective change of refractive index with carrier concentration. From (10), $\partial\left|R_{0}\right| / \partial N$ is positive and this is also true for $\partial\left|R_{1}\right| / \partial N$. Alternatively, it can be shown that 1) $\partial\left|R_{0,1}\right| / \partial \theta_{2}$ is negative as $\theta_{2}=0$ is the global maximum of $\left|R_{0,1}\right|$ [8], and 2) $\partial \theta_{2} / \partial N$ is also negative as indicated in (4), thus $\partial\left|R_{0,1}\right| / \partial N=$ $\partial\left|R_{0,1}\right| / \partial \theta_{2} \times \partial \theta_{2} / \partial N$ is positive. Both of the above argument shows that $\partial \alpha / \partial N$ has a negative value for index guiding lasers. In the following paragraph, the magnitude and sign of $\Gamma_{s}, \partial \Gamma / \partial N, \alpha_{s}$, and $\partial \alpha / \partial N$ are calculated for a VCSEL with index-guiding structure. We would attempt to see how these values vary in the VCSEL as we vary its core radius $W$, to verify if their relationships agree with predictions from our theoretical analysis presented here.

\section{Calculation of $\Gamma_{s}, \partial \Gamma / \partial N, \alpha_{s}$, and $\partial \alpha / \partial N$}

The values of $\Gamma_{s}, \partial \Gamma / \partial N, \alpha_{s}$, and $\partial \alpha / \partial N$ can be calculated for a schematic VCSEL given in Fig. 1. The device structure is similar to the fabricated VCSEL given in [11]. In our schematic VCSEL the top mirror is a p-type distributed Bragg reflector surrounded by a cladding layer of refractive index $n_{\text {clad }}$. The background effective refractive index of the core region (i.e., region including the top mirror and active layer) is equal to $n_{\text {core. }}$. Since the entire top reflector is confined within the core region, light diffraction is negligible there and the reflectivity $\left|R_{1}\right|$ is assumed a constant. The bottom mirror is an n-type distributed Bragg reflector formed by alternate layers (26 pairs) of $\mathrm{AlGaAs}$ and AlAs with quarter wavelength thickness. The values of $n_{\text {clad }}, n_{\text {core }},\left|R_{1}\right|$, and the refractive index distribution of the n-type Bragg reflector used in the calculation can be found in Table I.

Fig. 3 shows the variation of $\Gamma_{s}, \alpha_{s}, \partial \Gamma / \partial N$, and $\partial \alpha / \partial N$ with core radius $W$. The value of $\Gamma_{s}$ and $\partial \Gamma / \partial N$ are calculated [6] with the assumption that the small change of refractive index $\Delta n$ inside the active layer is related to $\Delta N$ by

$$
\Delta n=\frac{\partial n}{\partial N} \Delta N
$$

\begin{tabular}{|c|c|c|}
\hline Parameter & Symbol & Value \\
\hline wavelength & $\lambda_{0}$ & $0.98 \mu \mathrm{m}$ \\
\hline group velocity & $v_{g}$ & $0.83 \times 10^{10} \mathrm{~cm} / \mathrm{s}$ \\
\hline longitudinal optical confinement factor & $\Gamma_{\mathrm{z}}$ & 0.07 \\
\hline bimolecular carrier recombination coefficient & $\mathrm{B}_{\mathrm{sp}}$ & $1 \times 10^{-10} \mathrm{~cm}^{3} \mathrm{~s}^{-1}$ \\
\hline spontaneous emission factor & $\beta$ & $1 \times 10^{-4}$ \\
\hline active region thickness & d & $0.1 \mu \mathrm{m}$ \\
\hline background refractive index in core region & $\mathbf{n}_{\text {corc }}$ & 3.45 \\
\hline background refractive index in cladding region & $\mathbf{n}_{\mathrm{clad}}$ & 3.43 \\
\hline carricr lifetime in active layer & $\tau_{\mathrm{s}}$ & $3 \times 10^{-9} \mathrm{~s}$ \\
\hline carrier induced refractive index change & $\partial \mathrm{n} / \partial \mathrm{N}$ & $-6.816 \times 10^{-21} \mathrm{~cm}^{3}$ \\
\hline gain suppression factor & $\varepsilon$ & $1 \times 10^{-17} \mathrm{~cm}^{3}$ \\
\hline material loss & $\alpha_{m}$ & $10 \mathrm{~cm}^{-1}$ \\
\hline effective cavity length & $\mathrm{L}_{\text {eff }}$ & $0.7 \mu \mathrm{m}$ \\
\hline background temperature & $T_{r}$ & $300^{\circ} \mathrm{K}$ \\
\hline thermal conductivity & $\kappa$ & $0.45 \mathrm{Wcm}^{-1} \mathrm{C}^{-1}$ \\
\hline reflectivity of top Bragg reflector & $\left.\mid \mathbf{R}_{\mathbf{1}}\right\rceil$ & 0.997 \\
\hline characteristic temperature & $\mathrm{T}_{\mathrm{o}}$ & $30^{\circ} \mathrm{K}$ \\
\hline refractive index of AlAs dielectric layer & & 2.95 \\
\hline refractive index of $\mathrm{AlGaAs}$ dielectric layer & & 3.5 \\
\hline
\end{tabular}

TABLE I

PARAMETERS USED IN THE MOdel

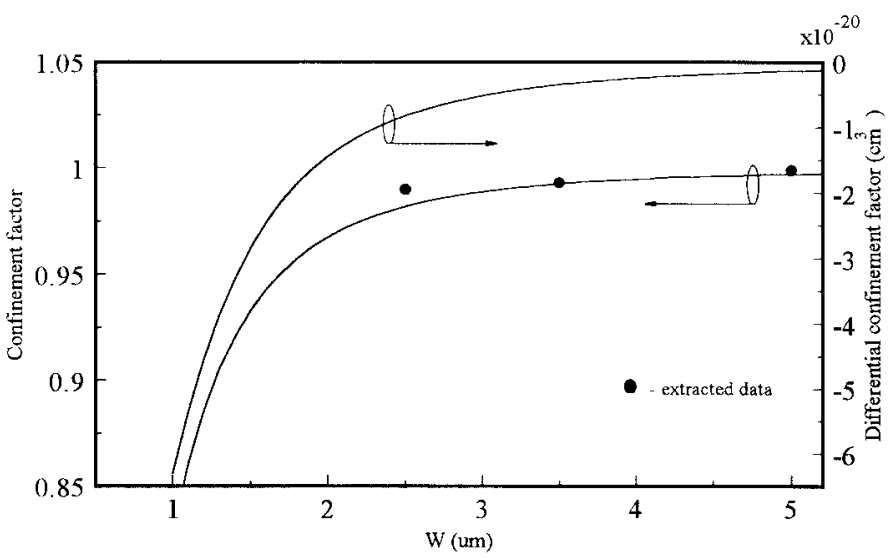

(a)

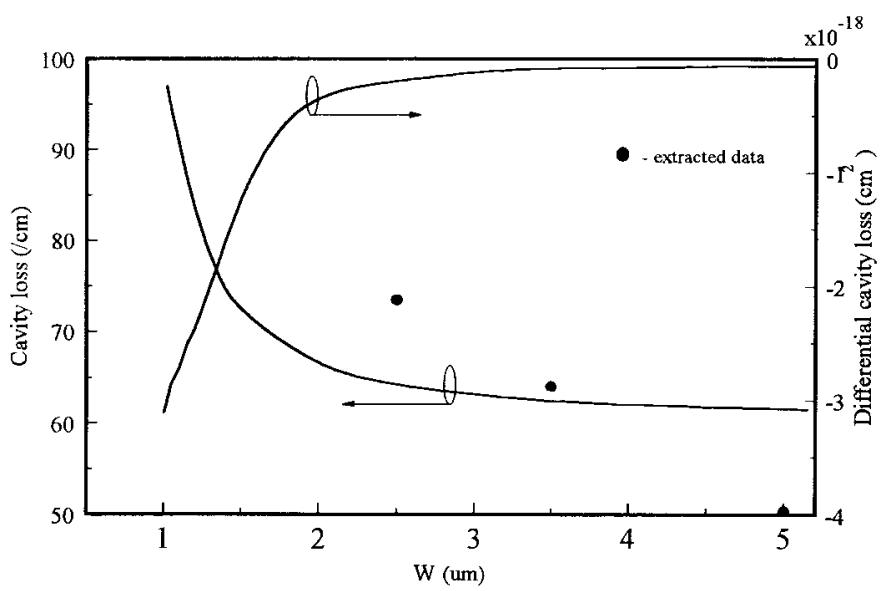

(b)

Fig. 3. (a) The variation of confinement factor $\left(\Gamma_{s}\right)$ and differential confinement factor $(\partial \Gamma / \partial N)$ with core radius, $W$. (b) The variation of cavity loss $\left(\alpha_{s}\right)$ and differential cavity loss $(\partial \alpha / \partial N)$ with core radius, $W$. 
where $\partial n / \partial N$ is the carrier induced index change. In addition, the values of $\alpha_{s}$ and $\partial \alpha / \partial N$ are calculated using (5) and (7) [5]. From the graphs of the calculated values in Fig. 3, it is clear that $\Gamma_{s}, \partial \Gamma / \partial N$, and $\partial \alpha / \partial N$ increase, but $\alpha_{s}$ decreases, with the increase of $W$. It can also be seen that both $\partial \Gamma / \partial N$ and $\partial \alpha / \partial N$ are negative for the whole range of $W$. As far as the general relationship between lateral loss parameters and core radius $W$ is concerned, the calculated results for this VCSEL agree readily with the analytical prediction. In Section III, these relationships are further confirmed by data extracted from measured characteristic of the fabricated VCSEL given in [11].

\section{Modulation ReSPONSE OF VCSEL WITH LATERAL LOSS EFFECTS}

\section{A. Rate-Equation Model with Lateral Loss Effects}

Using a rate-equation model, values of $\Gamma_{s}$ and $\alpha_{s}$ can be extracted from the measured light output versus current $(L-I)$ curves of the fabricated VCSEL given in [11]. The rate-equation model has been applied to the VCSEL for other investigations with success [4] and is given by

$$
\begin{aligned}
\frac{\partial P(t)}{\partial t} & =\nu_{g}(\Gamma(t) G(t)-\alpha(t)) P(t)+\beta B_{\mathrm{sp}} N^{2}(t) \\
\frac{\partial N(t)}{\partial t} & =\frac{I(t)}{q V}-\nu_{g} \Gamma(t) G(t) P(t)-\frac{N(t)}{\tau_{s}} \\
C_{\mathrm{th}} \frac{\partial T(t)}{\partial t} & =\left(P_{I V}(t)-P_{h \nu}(t)\right)-\kappa \frac{T(t)-T_{t}}{R_{\mathrm{th}}}
\end{aligned}
$$

where $P$ is the photon density, $N$ is the carrier concentration and $T$ is the effective temperature of laser cavity. In the photon rate equation, $G$ is the equivalent modal gain, $\nu_{g}$ is the group velocity, $\beta$ is the spontaneous emission factor and $B_{\mathrm{sp}}$ is the bimolecular carrier recombination factor. In the carrier rate equation, $\tau_{s}$ is the carrier lifetime, $I$ is the injection current and $V$ is the volume of the active layer. $\alpha$ and $\Gamma$ given in (12) and (13) take into account $\alpha_{s}$ and $\Gamma_{s}$ as well as $\partial \Gamma / \partial N$ and $\partial \alpha / \partial N$ for the consideration of lateral loss effects.

In the thermal rate equation, $T_{t}$ is the temperature at threshold, $\kappa$ is the thermal conductivity, $R_{\text {th }}$ and $C_{\text {th }}$ are the thermal resistance and capacitance, respectively, of the device. The output power $P_{h \nu}$ of the device is given by

$$
P_{h \nu}=\frac{1}{2} \nu_{g} h \nu\left(1-\left|R_{1}\right|^{2}\right) \pi W^{2} \Gamma_{z} P
$$

where $h$ is the Planck's constant and $\nu$ is the frequency of the lasing mode. The total input electrical power $P_{I V}$ is defined as

$$
P_{T V}=V_{F}(N) I
$$

where $V_{F}$ is the voltage across the active layer and is given by [15]

$$
\begin{aligned}
V_{F}=\frac{1}{q}\left[E_{g}+k_{B} T \cdot \log \left\{\left(\exp \left(N / N_{c}\right)-1\right)\right.\right. \\
\left.\left.\cdot\left(\exp \left(N / N_{v}\right)-1\right)\right\}\right]
\end{aligned}
$$

where $E_{g}\left(=1.519-5.408 \times 10^{-4} \times T^{2} /(T+204)\right)$ is the energy gap between the first quantized energy level of conduction and the valence bands of quantum wells active

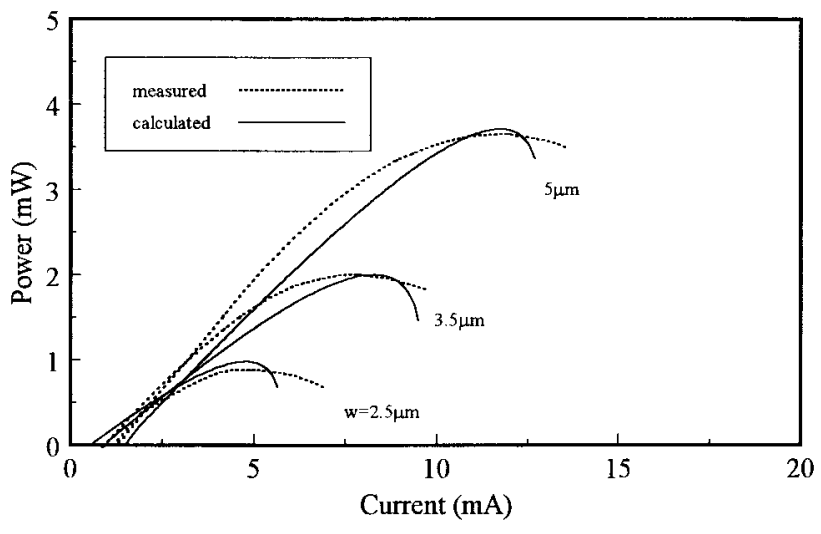

Fig. 4. Comparison of the measured and calculated light/current curves of VCSEL's with core radius equal to $2.5,3.5$, and $5 \mu \mathrm{m}$.

layer and $k_{B}$ is the Boltzmann constant. $N_{c}$ and $N_{v}$ are the effective conduction and valence edge density of states, respectively, and can be expressed as

$$
N_{c / v}=m_{c / h}^{*} k_{B} T / \pi \hbar^{2} L_{z}
$$

where $\hbar=h / 2 \pi, m_{c / h}^{*}(=0.0665 / 0.33196)$ is the effective mass of electron/holes and $L_{z}(=80 \AA)$ is the thickness of quantum wells.

The influence of nonlinear gain is considered in the model through the carrier and photon dependence of $G$ that is defined as

$$
G=\frac{G_{0}}{1+\varepsilon P}
$$

where $\varepsilon$ is the gain compression factor. $G_{0}$ is the unsaturated optical gain that is only dependent on the carrier concentration and can be expressed as

$$
G_{0}=\Gamma_{z} a_{N} \log \left(N / N_{0}\right)
$$

where $\Gamma_{z}$ is the longitudinal confinement factor, $a_{N}$ is the gain parameter and $N_{0}$ is the carrier concentration at transparency. In (20), the parameters, $a_{N}$ and $N_{0}$, are assumed to vary with temperature and their dependence are approximated by $a_{N}=$ $-46963.3+371.56 \times T-0.941029 \times T^{2}+7.99274 \times 10^{-4} \times$ $T^{3}, N_{0}=\left(2.723-0.02417 \times T+6.4786 \times 10^{-5} \times T^{2}\right) \times 10^{18}$ and $N$ in $G_{0}$ is normalized by $0.41 \times 10^{18} \mathrm{~cm}^{-3}$. The temperature dependence of the threshold current density $J_{\text {th }}$ (at $300 \mathrm{~K}$ ) is described by the Arrhenius-type relation [12]

$$
J_{\mathrm{th}}(T)=J_{o} \exp \left(\frac{T-300}{T_{o}}\right)
$$

where $J_{o}$ is the threshold current density at $300 \mathrm{~K}$ and $T_{o}$ is the characteristic temperature. $J_{0}$ can be expressed as $J_{0}=$ $q d N_{\text {th }} / \tau_{s}$ where $N_{\text {th }}$ is the threshold carrier concentration at $300 \mathrm{~K}$ and $d$ is the thickness of the active layer.

Fig. 4 shows the measured [11] and calculated light/current curves of the fabricated VCSEL at different $W$. The calculated data are obtained from (12)-(14) using (15)-(21) as the auxiliary equations. In the calculation, $\partial P / \partial t$ and $\partial N / \partial t$ and $\partial T / \partial t$ as well as $\partial \Gamma / \partial N$ and $\partial \alpha / \partial N$ are set to zero, $P, N$, and $T$ are then solved in a self-consistent manner. By matching the measured light/current curves given in [11] with 
TABLE II

Size Dependence of Optical Loss And Thermal Resistance of VCSEL'S

\begin{tabular}{c|c|c|c}
\hline $\begin{array}{c}\text { corc radius } \\
\mathrm{W}(\mu \mathrm{m})\end{array}$ & $\begin{array}{c}\text { lateral } \\
\text { confinement } \Gamma_{\mathrm{s}}\end{array}$ & $\begin{array}{c}\text { optical loss } \\
\alpha_{\mathrm{s}}\left(\mathrm{cm}^{-1}\right)\end{array}$ & $\begin{array}{c}\text { Thermal Resistance } \\
\mathrm{R}_{\mathrm{th}}\left(\mathrm{cm}^{-1}\right)\end{array}$ \\
\hline 2.5 & 0.990 & 73 & 7100 \\
\hline 3.5 & 0.993 & 64 & 4200 \\
\hline 5 & 0.999 & 50.3 & 2900 \\
\hline
\end{tabular}

the calculated curve, the values of $\Gamma_{s}, \alpha_{s}$, and $R_{\text {th }}$ used in the model are extracted from the experimental data. Extracted values of $\Gamma_{s}, \alpha_{s}$, and $R_{\mathrm{th}}$ are listed in Table II and other laser parameters used in the rate-equation model are given in Table I. The values of $\Gamma_{s}$ and $\alpha_{s}$ are also plotted in Fig. 3 (indicated as solid circle) for comparison with the calculated data from the VCSEL in Fig. 1. As we can see, $\Gamma_{s}$ and $\alpha_{s}$ extracted from the index-guided VCSEL in [11] exhibit precisely the same kind of variation with $W$ as would have predicted by the calculated results from the similar structured VCSEL in Section II above.

\section{B. Small-Signal Modulation Response of VCSEL: Second-Harmonic Distortion}

The rate-equation model can be simplified into two differential equations by using the quasistatic steady approximation on $T$ [4]. It is noted that the thermal lifetime of lasers is much longer than the oscillation period of $N$ and $P$. Therefore, the time derivative term in (14) can be ignored and the corresponding auxiliary thermal equation for quasi-steady state temperature $T_{s}$ is given by

$$
T_{s}=R_{\mathrm{th}} \cdot\left(P_{\Pi V}-P_{h \nu}\right) / \kappa+T_{t} .
$$

The small signal modulation response of VCSEL can be obtained by perturbation method [6]. This can be done by equating $I, N$, and $P$ with

$$
\begin{aligned}
I(t) & =I_{s}+\Delta I(t)=I_{s}+I_{1} e^{j \omega t} \\
N(t) & =N_{s}+\Delta N(t)=N_{s}+N_{1} e^{j \omega t}+N_{2} e^{j 2 \omega t} \\
P(t) & =P_{s}+\Delta P(t)=P_{s}+P_{1} e^{j \omega t}+P_{2} e^{j 2 \omega t}
\end{aligned}
$$

where $j=\sqrt{-1}, I_{s}$ is the bias current, $I_{1}$ and $\omega$ are the amplitude and modulation frequencies of the sinusoidal modulation current, respectively. The subscript $\mathrm{s}$ stands for the steady state. By substituting (23a)-(23c) into the rate equations (12) and (13), and after some manipulations, the AM response and SHD of VCSEL with lateral loss effects are given by

$$
\begin{aligned}
\frac{P_{1}}{I_{1}} & =\frac{\frac{\nu_{g} P_{s}}{q V} \Gamma_{s} \frac{\partial G_{\mathrm{eff}}}{\partial N}}{\left(\omega_{f}^{2}-\omega^{2}\right)+j \omega \nu_{g}\left(\kappa_{s}-\Gamma_{s} \frac{\partial G}{\partial P} P\right)} \\
\frac{P_{2}}{P_{1}} & =\frac{\left(2 j \omega+\nu_{g} \kappa_{s}\right) F(\omega)-\nu_{g} P_{s} \Gamma_{s} \frac{\partial G_{\mathrm{eff}}}{\partial N} E(\omega)}{\left(\omega_{f}^{2}-4 \omega^{2}\right)+2 j \omega \nu_{g}\left(\kappa_{s}-\Gamma_{s} \frac{\partial G}{\partial P} P_{s}\right)} P_{1} .
\end{aligned}
$$

The relaxation oscillation frequency $\omega_{f}$ is defined as

$$
\omega_{f}^{2}=\nu_{g}^{2} \Gamma_{s}\left(\alpha_{\mathrm{eff}} \frac{\partial G_{\mathrm{eff}}}{\partial N}-\kappa_{s} \frac{\partial G}{\partial P}\right) P_{s}
$$

where

$$
\begin{aligned}
\frac{\partial G_{\mathrm{eff}}}{\partial N} & =\frac{\partial G}{\partial N}+\frac{1}{\Gamma_{s}}\left(G_{s} \frac{\partial \Gamma}{\partial N}-\frac{\partial \alpha}{\partial N}\right) \\
\alpha_{\mathrm{eff}} & =\alpha_{s}\left(1-\frac{\varepsilon P_{s}}{\left.1+\varepsilon P_{s}\right)}\right) \\
\kappa_{s} & =\frac{1}{\nu_{g} \tau_{s}}+\left(G_{s} \frac{\partial \Gamma}{\partial N}+\Gamma_{s} \frac{\partial G}{\partial N}\right) P_{s} \\
\frac{\partial G}{\partial P} & =-\frac{\varepsilon \alpha}{\Gamma_{s}\left(1+\varepsilon P_{s}\right)} \text { and } \frac{\partial G}{\partial N} \frac{\Gamma_{z} a_{N}}{N_{0}} \exp \left\{-\frac{\alpha_{s}}{\Gamma_{s} \Gamma_{z} a_{N}}\right\} .
\end{aligned}
$$

In (25), $F(\omega)$ and $E(\omega)$ are defined as

$$
\begin{aligned}
E(\omega)= & \nu_{g} P_{s} \frac{\partial \Gamma}{\partial N} K(\omega)\left[\frac{\partial G}{\partial P}+\frac{\partial G}{\partial N} K(\omega)\right] \\
& +\nu_{g} \Gamma_{s}\left[\frac{\partial G}{\partial P}+\left(\frac{\partial G_{\mathrm{eff}}}{\partial N}+\frac{1}{\Gamma_{s}} \frac{\partial \alpha}{\partial N}\right) K(\omega)\right] \\
F(\omega)= & \nu_{g} P_{s} \frac{\partial \Gamma}{\partial N} K(\omega)\left[\frac{\partial G}{\partial P}+\frac{\partial G}{\partial N} K(\omega)\right] \\
& +\nu_{g} \Gamma_{s}\left[\frac{\partial G}{\partial P}+\frac{\partial G_{\mathrm{eff}}}{\partial N} K(\omega)\right]
\end{aligned}
$$

where

$$
K(\omega)=\left(\nu_{g} P_{s} \Gamma_{s} \frac{\partial G_{\mathrm{eff}}}{\partial N}\right)^{-1} \times\left(j \omega-\nu_{g} \Gamma_{s} P_{s} \frac{\partial G}{\partial P}\right)
$$

As we can see, $E(\omega)$ and $F(\omega)$ are only different by an extra term, $\nu_{g}(\partial \alpha / \partial N) K(\omega)$. In the above derivation, we have ignored spontaneous emission term.

Fig. 5 shows the SHD of the fabricated VCSEL, calculated from (25), at $W=3.5$ and $W=5 \mu \mathrm{m}$. The device is biased at around $I_{s}=4 \mathrm{~mA}$ with steady state output power of 1 $\mathrm{mW}$ and undergoes small signal current modulation $(\sim 0.5$ $\mathrm{mA}$ ) of frequency $\omega$. In the calculations, the values of $\Gamma_{s}, \alpha_{s}$, and $R_{\text {th }}$ are obtained from Table II. At each $W$ value, two curves are obtained. In the first, both $\partial \Gamma / \partial N$ and $\partial \alpha / \partial N$ are set to zero to simulate the SHD response without lateral loss effects. In the second, $\partial \Gamma / \partial N$ and $\partial \alpha / \partial N$ are assigned their most probable values (At $W=3.5 \mu \mathrm{m}, \partial \Gamma / \partial N$ and $\partial \alpha / \partial N$ are equal to $-0.4 \times 10^{-20} \mathrm{~cm}^{3}$ and $-0.1 \times 10^{-18}$ $\mathrm{cm}^{2}$. At $W=5 \mu \mathrm{m}, \partial \Gamma / \partial N$ and $\partial \alpha / \partial N$ are equal to $-0.02 \times 10^{-20} \mathrm{~cm}^{3}$ and $-0.03 \times 10^{-18} \mathrm{~cm}^{2}$ ) according to Fig. 3 to simulate the response with lateral loss. In the figure, the dotted and dashed lines represent the VCSEL SHD with and without lateral loss effects, respectively. It is observed that, at low modulation frequencies, SHD with lateral loss effects is much higher than that without lateral loss. For modulation frequencies below a cutoff frequency $\omega_{\text {cutoff }}$ (e.g., $\omega<0.6 \times 10^{8} \mathrm{~Hz}=\omega_{\text {cutoff }}$ for the device with $W=3.5 \mu \mathrm{m}$ ), SHD is very much independent of $\omega$. For modulation above 


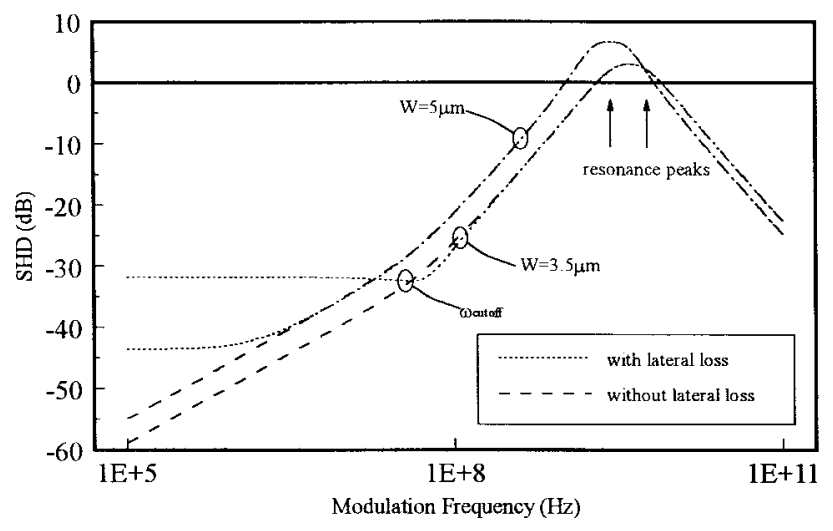

Fig. 5. Second-harmonic distortion of VCSEL's with core radius of 3.5 and $5 \mu \mathrm{m}$. The steady-state output power of VCSEL's is set to $1 \mathrm{~mW}$.

the cutoff, $\partial \Gamma / \partial N$ and $\partial \alpha / \partial N$ have little effect on SHD as the SHD curves with and without lateral loss essentially overlap. Furthermore, When core radius $W$ is increased from 3.5 to $5 \mu \mathrm{m}$, at no lateral loss, SHD is uniformly increased throughout all modulation frequencies up to $\omega_{f}$; in the more realistic situation where lateral loss is present, the SHD is decreased, and the cutoff frequency is lowered resulting in a narrower modulation bandwidth.

From the above numerical analysis, it is clear that the two lateral loss parameters, either $\partial \Gamma / \partial N$ or $\partial \alpha / \partial N$, or both, have profound effect on SHD only at low modulation frequencies. It is interesting then to further investigate how each of the lateral loss parameter affects the SHD. This can be done analytically by simplifying the expression for SHD in (25) at low modulation frequencies. Hence, for $\omega \ll \omega_{f}$, (24) and (25) is reduced to

$$
\begin{aligned}
& \frac{P_{1}}{I_{1}} \approx \frac{\frac{\nu_{g} P_{s}}{q V} \Gamma_{s} \frac{\partial G_{\mathrm{eff}}}{\partial N}}{\omega_{f}^{2}} \\
& \frac{P_{2}}{P_{1}} \approx \frac{\nu_{g} \kappa_{s} F(\omega)-\nu_{g} P_{s} \Gamma_{s} \frac{\partial G_{\mathrm{eff}}}{\partial N} E(\omega)}{\omega_{f}^{2}} P_{1} .
\end{aligned}
$$

For large $P_{s}\left(\right.$ say $\left.5 \times 10^{15} \mathrm{~cm}^{-3}\right)$, the value $\Gamma_{s}(\partial G / \partial N) P_{s}$ of is roughly equal to $0.25 \mathrm{~cm}^{-1}$ which is larger than $1 / \nu_{g} \tau_{s}\left(=0.04 \mathrm{~cm}^{-1}\right)$. Thus $\left(1 / \nu_{g} \tau_{s}\right)+P_{s} G_{s}\left(\partial G_{\text {eff }} / \partial N\right) \ll$ $P_{s} \Gamma_{s}(\partial G / \partial N)$, therefore, $\kappa_{s} \approx P_{s} \Gamma_{s}\left(\partial G_{\text {eff }} / \partial N\right)$. The numerator of (29) becomes $\nu_{g} P_{s} \Gamma_{s}\left(\partial G_{\text {eff }} / \partial N\right) \cdot \nu_{g}(\partial \alpha / \partial N)$ $K(\omega)$ where $K(\omega) \approx(\partial G / \partial P)\left(\partial G_{\text {eff }} / \partial N\right)^{-1}$ at low modulation frequencies. Equation (29) can then be reduced to

$$
\frac{P_{2}}{P_{1}} \approx \nu_{g} P_{s} \Gamma_{s} \cdot \nu_{g} \frac{\partial \alpha}{\partial N} \frac{\partial G}{\partial P} \frac{P_{1}}{\omega_{f}^{2}}
$$

Thus the expression for SHD is reduced to a form independent of $\omega$ conforming to the leveling SHD region in Fig. 5 for modulation frequencies below $\omega_{\text {cutoff. }}$ From (24) and (26), $P_{1}$ and $\omega_{f}$ are related to $\partial \Gamma / \partial N$ and $\partial \alpha / \partial N$ through $\left(\partial G_{\text {eff }} / \partial N\right)=(\partial G / \partial N)+\left(1 / \Gamma_{s}\right)\left(G_{s}(\partial \Gamma / \partial N)-\right.$ $(\partial \alpha / \partial N))$. In a typical VCSEL, the true values of $G_{s} \partial \Gamma / \partial N$ and $\partial \alpha / \partial N$ (see Fig. 3) are 100 times less than $\partial G / \partial N$ such that $\left(\partial G_{\mathrm{eff}} / \partial N\right) \approx(\partial G / \partial N)$, indicating that $P_{1}$ and $\omega_{f}$ are essentially independent of $\partial \Gamma / \partial N$ and $\partial \alpha / \partial N$. The term $(\partial G / \partial P)=-\left(\varepsilon \alpha_{s} / \Gamma_{s}\left(1+\varepsilon P_{s}\right)\right)$ does not involve $\partial \Gamma / \partial N$ and $\partial \alpha / \partial N$. Thus (30) describes that SHD is directly proportional to $|\partial \alpha / \partial N|$ at low modulation frequencies. $\partial \Gamma / \partial N$ has no effect here.

\section{DiscuSSION AND CONCLUSION}

From the above, the effect of lateral loss on SHD due to differential cavity loss $\partial \alpha / \partial N$ kicks in at modulation frequencies below $\omega_{\text {cutoff }}$ (e.g., $600 \mathrm{MHz}$ in the device with $W=3.5 \mu \mathrm{m})$. This is a source of noise in optical systems. A common approach used for transmitting signal is by intensity modulation and two major problems exist in such a scheme: the noise levels produced are high and the full bandwidth of the optical system cannot be used [13]. In Fig. 5, the device at $W=5 \mu \mathrm{m}$ exhibits about $-45 \mathrm{~dB}$ SHD, and the device at $W=3.5 \mu \mathrm{m}$ exhibits a little over $-30-\mathrm{dB}$ SHD. Such levels of intensity noise are pretty much in line with those that are found in edge emitting devices [14]. However, VCSEL allows for a much larger modulation bandwidth. Our study show that paying careful consideration to lateral loss effects coupled with smaller core size design, the VCSEL devices can offer more promising solution than edge emitting laser device to the problems that exist in the intensity modulation systems. As observed in our simulation, using a larger core radius $\mathrm{W}$ reduces SHD, but in the expense of modulation bandwidth, since the cutoff frequency $\omega_{\text {cutoff }}$ would be lowered. The increase in SHD at smaller $W$ is due to a combination of increase in $\Gamma_{s}, \alpha_{s}$, and $\partial \alpha / \partial N$ as $W$ decreases. Thus, it becomes more critical to consider lateral loss effects and means of minimizing SHD, when designing VCSEL with small core size. For example, it can be observed from (30) that the dependence of SHD on $\partial \alpha / \partial N$ can be reduced by decreasing $\partial G / \partial P$, via the reduction of gain compression factor $\varepsilon$. Furthermore, the direct relationship between SHD and $\partial \alpha / \partial N$ in (30) assumes that the photon density $P_{s}$ is large. Lowering the output intensity can, therefore, also reduce the dependence of SHD on $\partial \alpha / \partial N$.

In conclusion, the influence of lateral loss effects on the SHD of VCSEL is investigated theoretically. Two parameters, $\partial \Gamma / \partial N$ and $\partial \alpha / \partial N$, are defined for the consideration of selffocusing and diffraction loss in VCSEL under small signal modulation. A simple rate-equation model is developed and the analytical expression of SHD is derived. It is shown that $|\partial \alpha / \partial N|$ is the dominant factor that induces SHD at low modulation frequencies, especially for devices with small core radius.

\section{REFERENCES}

[1] T. E. Darcie and R. S. Tucker, "Intermodulation and harmonic distortion in InGaAsP lasers," Electron. Lett., vol. 21, pp. 665-666, 1985.

[2] G. Morthier, F. Libbrecht, K. David, P. Vankwikelberge, and G. Baets, "Theoretical investigation of the second order harmonic distortion in the AM response of $1.55 \mu \mathrm{m}$ F-P and DFB lasers," IEEE J. Quantum Electron., vol. 27, pp. 1990-2002, Aug. 1991.

[3] D. Tauber, G. Wang, R. S. Geels, J.E. Bowers, and L. A. Coldern, "Large and small signal dynamics of vertical cavity surface emitting lasers," Appl. Phys. Lett., vol. 62, pp. 325-327, 1993. 
[4] S. F. Yu, W. N. Wong, P. Shum, and E. H. Li, "Theoretical analysis of modulation response and second harmonic distortion in vertical cavity surface emitting lasers," IEEE J. Quantum Electron., vol. 32, pp. 2139-2147, Dec. 1996.

[5] S. F. Yu and E. H. Li, "Influence of lateral field on the relaxation oscillation frequency of semiconductor lasers," IEEE J. Quantum Electron., vol. 32, pp. 1-3, Jan. 1996.

[6] S. F. Yu, "Analysis and design of vertical cavity surface emitting lasers for self-sustained pulsation operation," IEEE J. Quantum Electron., vol. 34, pp. 497-505, Mar. 1998.

[7] E. Hechit, Optics, 2nd ed., Addison-Wesley, 1987.

[8] Y. Yeh, Optical Waves in Layered Media. New York: Wiley, 1988, ch. 6.

[9] R. R. Burton, M. S. Stern, P. C. Kendall, and P. N. Robson, "Modeling of diffraction in pillar vertical cavity surface emitting lasers with embedded Bragg layers," Opt. Quantum Electron., vol. 28, pp. 1677-1684, 1996.

[10] J. K. Butler and J. Zoroofchi, "Radiation fields of GaAs-(AlGa)As Injection lasers," IEEE J. Quantum Electron., vol. pp. 809-815, 1974.

[11] J. W. Scott, R. S. Geels, S. W. Corzine, and L. A. Coldren, "Modeling temperature effects and spatial hole burning to optimize vertical cavity surface emitting laser performance," IEEE J. Quantum Electron., vol. 29, pp. 1295-1308, 1993.

[12] N. K. Dutta, J. Lopata, D. L. Sivco, and A. Y. Cho, "Temperature dependence of threshold of strained quantum well lasers," Appl. Phys. Lett., vol. 58, no. 11, pp. 1125-1127, 1991.

[13] J. Singh, Optoelectronics-An Introduction to Materials and Devices. New York: McGraw Hill, 1996, ch. 10.

[14] G. Keiser, Optical Fiber Communications. New York: McGraw Hill, 1991, ch. 4.

[15] R. S. Zory, Ed., Quantum Well Lasers. New York: Academic, 1993, ch. 1 .

P. C. Chui, photograph and biography not available at the time of publication.

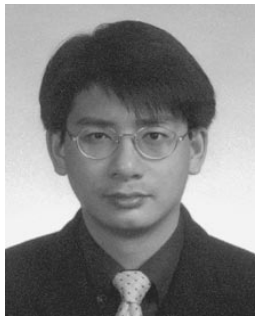

S.-F. Yu received the degree of B.E. (first class Honors) degree in electronic engineering from London University, University College, U.K., in 1990 and the degree of Doctor of Philosophy in optoelectronics engineering from Cambridge University, Robinson College, U.K., in 1993.

He joined the Department of Electronic Engineering, Sha Tin Technical Institute, Hong Kong, as a part-time Lecturer in 1993. In 1994, he joined the Department of Electrical and Electronic Engineering, the University of Hong Kong, where he was a Lecturer. Since 1996, he has been an Assistant Professor in the same department of the University of Hong Kong. His main research interest includes the fundamental study, optimization and the practical applications of semiconductor lasers. He currently conducts the development of high performance semiconductor lasers using diffused quantum-well materials for the application in long haul optical fiber communication systems. Furthermore, he involves in the development of novel mid-infrared vertical cavity lasers using quantum cascade architecture and their applications in the measurement of gas and liquid concentrations. He also studies the possibilities of using organic materials to fabricate integrated optoelectronics circuit including lasers, modulators and photodetectors for the industrial applications. He has published over 70 international technical papers including invited conference and journal papers as well as two book chapters.

Dr. Yu was awarded the traditional Departmental Prize by the Department of Electronics and Electrical Engineering, University College London in the final year degree examination. He was a Fellow and Honorary Scholar of Cambridge Commonwealth Trust Society. He also held a Croucher Foundation scholarship and an overseas research student award while studying for the doctoral program. Dr. Yu is a member of the SPIE. He is also in the executive committee, and is the seminar program chair of the seminar and meeting committee of SPIE Hong Kong Chapter. His biography has published in Who's Who In Science And Engineering (4th ed., Marquis), Dictionary of International Biography (25th ed.), and the International Directory of Distinguished Leadership (17th ed.). 